\title{
Quasi-Periodic Lattices: pattern matters too
}

\author{
Audrey Somera ${ }^{\mathrm{a}, *}$, Martin Poncelet ${ }^{\mathrm{b}}$, Nicolas Auffray $^{\mathrm{c}}$, Julien Réthoréa \\ ${ }^{a}$ Research Institute in Civil Engineering and Mechanics (GeM), Centrale Nantes, Université de Nantes, CNRS, UMR 6183, 1 \\ rue de la Noë, 44321 Nantes Cedex 3, France \\ ${ }^{b}$ Université Paris-Saclay, ENS Paris-Saclay, CNRS, LMT - Laboratoire de Mécanique et Technologie, France \\ ${ }^{c}$ MSME, Univ Gustave Eiffel, CNRS UMR 8208, F-77474 Marne-la-Vallée, France
}

\begin{abstract}
Two kinds of behaviour are commonly distinguished among porous architectured materials: stretchingdominated and bending-dominated ones. One or the other is favoured depending on the mechanical application. This property has been widely studied for random foams and periodic lattices, but has not yet been considered for quasi-periodic ones. Generally speaking, few studies have investigated their mechanical behaviour, and when this has been done, the impact of the elementary pattern shape has not been considered. In this paper we propose a classification of quasi-periodic structures depending on their dominance type by performing numerical simulations on mesoscopic models, and analysing the proportions of the strain energy stored in stretching and bending. As a result, three different dominance behaviours can be distinguished: completely stretching or bending dominated and varying dominance. Belonging to one or the other category depends mainly on the elementary pattern shape, and thus so does the overall mechanical behaviour.
\end{abstract}

Keywords: Quasi-periodic lattices, Mechanical properties, Elastic behavior, Finite element analysis, Architectured materials

The rise of new means of production such as additive manufacturing has led to an increasing interest in architectured materials because of their good specific properties $[1,2,3]$. Concerning porous architectured materials, they can be classified into two different categories: (1) random materials as foams and (2) periodic materials like honeycomb lattices. Each category has its strength and its drawbacks. Foams are easy to produce and have isotropic properties in average, but there is randomness in their manufacturing processes, and their properties can vary from one sample to another, and even within a sample. On the contrary, the mesostructure of periodic lattices is deterministic and their study can be conducted on a single unit cell, although they tend to be more anisotropic [4] and to have low fracture toughness [5, 6]. A way to combine the advantages of both categories can be to consider quasi-periodic lattices. Indeed, they are highly deterministic structures that possess long range order in the same time as they appear to be locally disorganized [7]. In addition, they can exhibit a global isotropic behaviour $[8,9]$ and a better failure resistance than periodic lattices [6].

It has been shown that foams and periodic architectured materials can be classified into two different categories: so-called stretching-dominated and bending-dominated structures [10, 1, 11, 5, 12]. The assignment depends on the way the structure deforms when subjected to a mechanical loading. One or the other behaviour is favoured depending on the situation. For structural applications stretching-dominated materials are usually favoured as they have a higher stiffness and compressive strength for a given density. For example, for a density of 0.1 , this kind of pattern will be 10 times stiffer and 3 times stronger than a bending-dominated one $[10,1]$. Conversely, when seeking to optimise the energy dissipation of the structure or designing auxetic materials, bending-dominated materials are preferred [13,14]. To the best of the authors' knowledge, the dominance type of quasi-periodic structures has not yet been investigated. Very few studies are dedicated

\footnotetext{
* Corresponding author

Email addresses: audrey.somera@ec-nantes.fr (Audrey Somera), martin.poncelet@ens-paris-saclay.fr (Martin Poncelet), nicolas.auffray@univ-eiffel.fr (Nicolas Auffray), julien.rethore@ec-nantes.fr (Julien Réthoré)
} 
to their mechanical properties, and the role of the elementary pattern is usually not analyzed, favouring the study of the influence of the symmetry class $[15,9]$.

In this paper, the role of the pattern of quasi-periodic lattices on their mechanical behaviour is investigated, in particular its influence on the energy dominance type of the obtained structure. The impact of its symmetry class is also considered. First, a set of different periodic and quasi-periodic lattices having different elementary patterns and belonging to different symmetry classes are selected. Tensile test simulations are performed on 2D mesoscopic models. The proportions of the strain energy stored in the form of tension/compression and bending, depending on the beam slenderness, are then estimated globally and locally. Finally, the different patterns are classified according to their dominance type.

A lattice is periodic when generated with well-defined translations of an elementary pattern and so it is perfectly ordered locally and globally. Conversely, quasi-periodic lattices apparently have no local order but they are actually both highly organised structures and possess long range order, as indicated by their sharp diffraction diagram $[7,16,17]$. They are a generalisation of periodic structures for which the rotational invariance is not compatible with the translational one. The most famous examples of such lattices are the Penrose and Ammann-Beenker tilings [18, 19, 20, 21].

In the present contribution, the patterns have been chosen to produce isotropic effective elastic behaviour. What differs between them are the local deformation mechanisms, the nature of which is decisive for the nonlinear behaviour of the resulting lattice material. Our objective here is not to identify homogenised elastic parameters, but rather to go beyond homogenisation and study the local response of the mesostructure. Thus, this paper focuses only on 2D patterns, and the symmetry classes are chosen to obtain transverse isotropic first order linear elastic behaviours by homogenisation [22]. Quasi-periodic lattices with various geometries of elementary patterns and belonging to diverse symmetry classes are considered. Some periodic patterns are also included for comparison purposes. The different patterns and the nomenclature used in this paper are given in Figure 1.

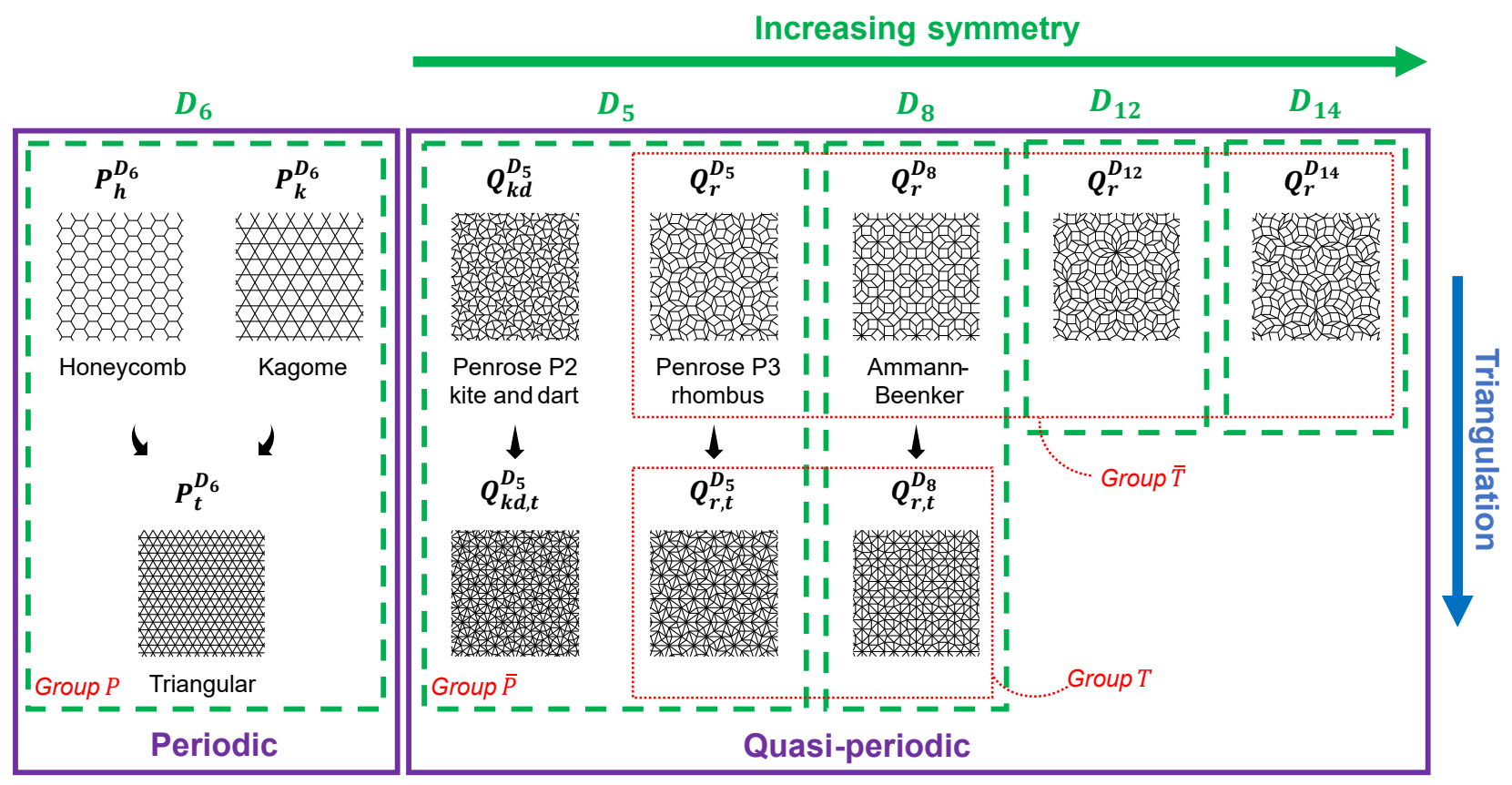

Figure 1: Patterns studied and nomenclature used.

One can note that all the patterns belong to a $\left[D_{k}\right]$ type symmetry class. For periodic lattices it means that the unit cell pattern is invariant by rotation of $\frac{2 \pi}{k}$ and possesses $k$ mirror lines [23]. For quasi-periodic ones, it means that the Fourier transform of the lattice has these types of symmetry [17, 24]. 
To study the influence of the elementary pattern geometry and of the symmetry class independently, the lattices have been grouped into the following categories:

- patterns belonging to the same symmetry class but having different geometries of elementary patterns

- Group $P: P_{h}^{D_{6}}, P_{t}^{D_{6}}$ and $P_{k}^{D_{6}}$ patterns ${ }^{1}$ which are periodic. They belong to the $\left[D_{6}\right]$

- Group $\bar{P}: Q_{k d}^{D_{5}}, Q_{k d, t}^{D_{5}}, Q_{r}^{D_{5}}$ and $Q_{r, t}^{D_{5}}$ patterns ${ }^{2}$ which are quasi-periodic. They belong to the $\left[D_{5}\right]$ symmetry class.

- patterns having similar geometries of elementary patterns, but belonging to different symmetry classes

- Group $T: Q_{r, t}^{D_{5}}$ and $Q_{r, t}^{D_{8}}$ whose elementary patterns are triangle-shaped.

- Group $\bar{T}: Q_{r}^{D_{5}}, Q_{r}^{D_{8}}, Q_{r}^{D_{12}}$ and $Q_{r}^{D_{14}}$ whose elementary patterns are rhombus-shaped.

Maxwell's criterion $M$ is usually used to classify periodic lattice structures [26, 1, 11, 5]. Its 2D expression is:

$$
M=b-2 j+3 \quad \text { with }\left\{\begin{array}{l}
b: \text { the number of pin-jointed struts; } \\
j: \text { the number of frictionless nodes. }
\end{array}\right.
$$

If $M$ is greater than or equal to 0 the pattern is said to be stretching-dominated, otherwise it is bendingdominated. The study of the average connectivity $Z$ of a large pin-jointed framework enables to easily estimate $M: Z=4$ implies $M=0$ in 2D [1]. Even if designed for articulated structures, the criterion can be applied for slender clamped beams. For periodic lattices, the criterion is commonly computed on the unit cell.

However, this criterion is not a sufficient condition to determine whether a structure will be stiff, even for periodic ones. In fact, it can take into account struts that do not contribute to global rigidity. Furthermore, it does not consider a potential dependence of the stiffness on the loading direction. Actually, some periodic structures exhibit a stretching-dominated behaviour while their criterion indicates a bending-dominated one [12]. The relevance of the criterion for quasi-periodic structures can therefore be questioned. The authors consequently favoured another method to establish the dominance type.

A tensile test simulation is performed on $150 \times 50 \mathrm{~mm}$ rectangular specimens, of which the average beam length ${ }^{3}$ for each pattern is chosen equal to $3.5 \mathrm{~mm}^{4}$. 2D mesoscopic models were used: a linear elastic EulerBernoulli model is associated to each beam of the lattice. The boundary conditions are given in Figure 2. A unitary load is distributed on the nodes belonging to the upper boundary. The lower boundary has been chosen so that it corresponds to a symmetry plane of the pattern and boundary conditions corresponding to this symmetry condition are applied. Simulations were run for beam slenderness between 10 and 500 to study its influence on the strain modes. The geometry of the specimens was chosen so that they can possibly be manufactured, at least for small slenderness.

The type of dominance of a pattern is linked to its deformation mechanisms. The predominant kind of deformation in a sample can be determined from the analysis of its corresponding strain energy. Let $U_{t o t}$ be the total strain energy of the sample. Considering beam elements, this energy can be divided into two parts: the tension-compression and bending contributions, called respectively $U_{S}$ and $U_{B}$. By normalising $U_{S}$ and $U_{B}$ by the total strain energy $U_{t o t}$, one can easily determine which contribution prevails. Beams close to boundary conditions are not considered for the energy calculation to avoid localisation and numerical singularities effects (cf. Figure 2). These energies are also computed locally to achieve a spatial analysis and to determine which type is stored in each beam.

\footnotetext{
${ }^{1} P_{t}^{D_{6}}$ can be obtained from both $P_{h}^{D_{6}}$ or $P_{k}^{D_{6}}$ by triangulation.

${ }^{2} Q_{k d, t}^{D_{5}}$ (resp. $Q_{r, t}^{D_{5}}$ ) is generated by the triangulation of $Q_{k d}^{D_{5}}$ (resp. $Q_{r}^{D_{5}}$ ) using the classical cut of kite and dart and rhombus patterns by triangles [25]. $Q_{r, t}^{D_{8}}$ is generated with an equivalent arbitrary cutting of the elementary patterns.

${ }^{3} Q_{k d}^{D_{5}}, Q_{k d, t}^{D_{5}}, Q_{r, t}^{D_{5}}$ and $Q_{r, t}^{D_{8}}$ patterns have beams of two slightly different lengths.

${ }^{4}$ The order of magnitude of the scale separation ratio $\eta$ defined as the sample width divided by the average beam length is approximately estimated to 14 .
} 


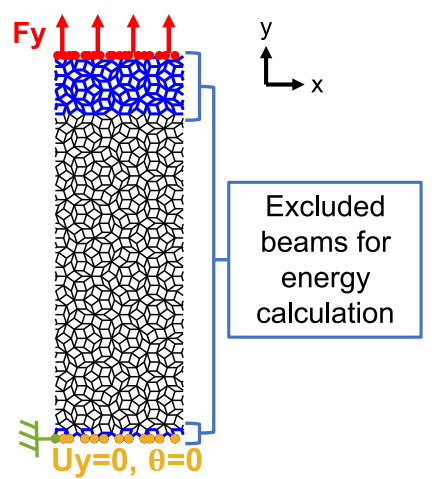

Figure 2: Boundary conditions applied for the simulations and beams excluded for the energy computations (in blue).

Figure 3 gives the evolution of the proportion of global strain energy stored as tensile energy $\frac{U_{S}}{U_{t o t}}$ for different specimens as a function of the beam slenderness. Figures 3.a and 3.b provide the results for groups $P$ and $\bar{P}$ respectively.

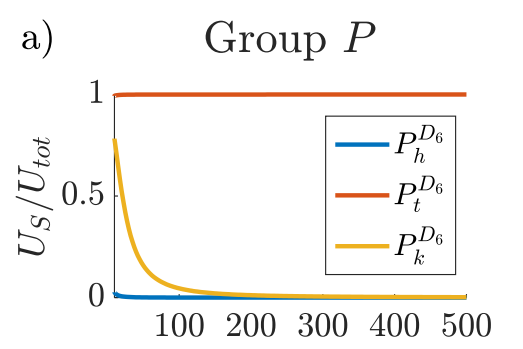

b) $\quad$ Group $\bar{P}$
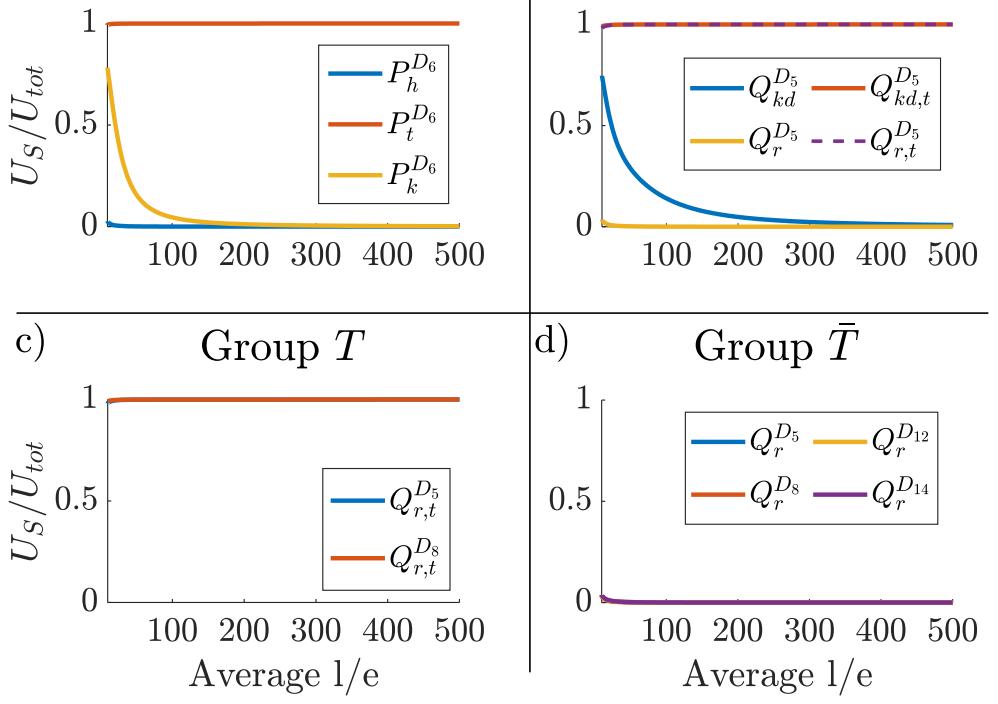

Figure 3: Evolution of the tensile energy ratio with the average slenderness for the different specimens.

Three kinds of behaviour are observed: two unchanging dominance and one varying dominance with the slenderness. The $Q_{k d, t}^{D_{5}}, Q_{r, t}^{D_{5}}$ and $P_{t}^{D_{6}}$ specimens have always a stretching dominated behaviour while the $Q_{r}^{D_{5}}$ and $P_{h}^{D_{6}}$ patterns always a bending dominated one. One observes on Figure 4 that the spatial energy distribution seems independent on the slenderness for those 2 cases. Moreover, almost all the beams store the same kind of energy, tensile or bending one in accordance with the global energy. Finally, the strain energy appears to be almost uniformly distributed throughout the specimen.

On the contrary, $Q_{k d}^{D_{5}}$ and $P_{k}^{D_{6}}$ patterns exhibit a dominating behaviour that depends on the slenderness. For small slenderness, the samples are stretching dominated, and for higher values they are bending dominated. Then, there is a significant change in the spatial distribution and type of energy stored. Contrary to the patterns with an unchanging dominance type, for a high slenderness of the beams, one observes that the distribution of strain energy is now highly heterogeneous and that almost all the beams deform in bending. Then, for low slenderness, even if the samples are globally stretching dominated, the spatial distribution is partitioned with beams storing all kinds of energies: tensile, bending or a mix of both.

It is worth noting that lattices that are consistently stretching-dominated always have an estimated Maxwell criterion higher than zero, see Table 1 . For periodic patterns a trend seems to be emerging as $P_{k}^{D_{6}}$ 
has a Maxwell criterion equal to zero and a varying dominance, and $P_{h}^{D_{6}}$ is bending dominated with $M$ lower than zero. However, $Q_{k d}^{D_{5}}$ and $Q_{r}^{D_{5}}$ have both a zero Maxwell criterion but exhibit different behaviours, respectively a varying dominance and a bending one. Thus, the idea that Maxwell's criterion is a necessary but not sufficient condition is confirmed for quasi-periodic patterns.

\begin{tabular}{|c|c||c|c|c||c|c|c|c|}
\hline \multirow{2}{*}{ Type } & Group & \multicolumn{3}{|c||}{$P$} & \multicolumn{4}{c|}{$\bar{P}$} \\
& Pattern & $P_{h}^{D_{6}}$ & $P_{k}^{D_{6}}$ & $P_{t}^{D_{6}}$ & $Q_{r}^{D_{5}}$ & $Q_{k d}^{D_{5}}$ & $Q_{r, t}^{D_{5}}$ & $Q_{k d, t}^{D_{5}}$ \\
\hline \hline \multirow{3}{*}{ Estimation } & $Z$ & 3 & 4 & 6 & 4 & 4 & 6 & 6 \\
& $M$ & $<0$ & 0 & $>0$ & 0 & 0 & $>0$ & $>0$ \\
& Dominance & Bend & Stretch & Stretch & Stretch & Stretch & Stretch & Stretch \\
\hline Observation & Dominance & Bend & Vary & Stretch & Bend & Vary & Stretch & Stretch \\
\hline
\end{tabular}

Table 1: Average connectivity ${ }^{5}$, Maxwell criterion and dominances estimated and observed for the patterns of groups $P$ and $\bar{P}$.

Figures 3.c and 3.d provide the results for groups $T$ and $\bar{T}$ respectively. First, none of the tested patterns of these groups has varying energy type. Second, the symmetry class of the patterns seems to have no influence on the dominance type, at least as far as unchanging dominance type patterns are concerned. Regarding $Q_{r}^{D_{8}}$ and $Q_{r}^{D_{5}}$ patterns, spatial distributions are comparable.

The energetic dominance type of quasi-periodic lattices was investigated using a numerical method: tensile test simulations were performed on 2D mesoscopic models and the proportions of strain energy stored by tension/compression and bending modes were computed globally and locally. It is subsequently possible to classify quasi-periodic architectured materials into three categories:

- Completely stretching-dominated: the material is stretching-dominated no matter the slenderness of its beams and almost all of them deform in tension or compression;

- Completely bending-dominated: the material is bending-dominated no matter the slenderness of its beams and almost all of them deform in bending;

- Varying dominance: the type of dominance varies with the slenderness and there may not be a common type of deformation of their beams.

This categorisation is also relevant for periodic structures. As for these ones, the Maxwell criterion is a necessary but not sufficient condition to obtain stretching-dominated quasi-periodic lattices. In the present study, the cases were selected so as to exhibit a transverse isotropic Cauchy elasticity, in order to have comparable behaviours. Within this investigation field, the belonging to one or the other category of dominance seems to be independent of the symmetry class of the lattice and depends only on the shape of the elementary patterns considered. Thus, the elementary pattern shape must be taken into account when studying the mechanical behaviour of quasi-periodic lattices. This is even more important when dealing with macroscopic non-linear lattice properties, such as plasticity, damage, or failure modes. In conclusion, for quasi-periodic lattices, a simple formula like Maxwell's cannot be obtained. In the case of periodic lattices, it is indeed well known that such a formula only gives an idea of the dominance mode of the lattice. As we show here, the structural analysis procedure is necessary for periodic lattices and even more so for quasi-periodic structures of any type to evaluate their dominance. For quasi-periodic lattices, a first idea of the dominance mode is obtained by calculating the average connectivity of the elementary patterns, but this result will have to be confirmed numerically as detailed here.

\footnotetext{
${ }^{5}$ For quasi-periodic patterns, $Z$ corresponds to the limit value when increasing the framework size, and thus making the influence of the boundary conditions negligible.
} 


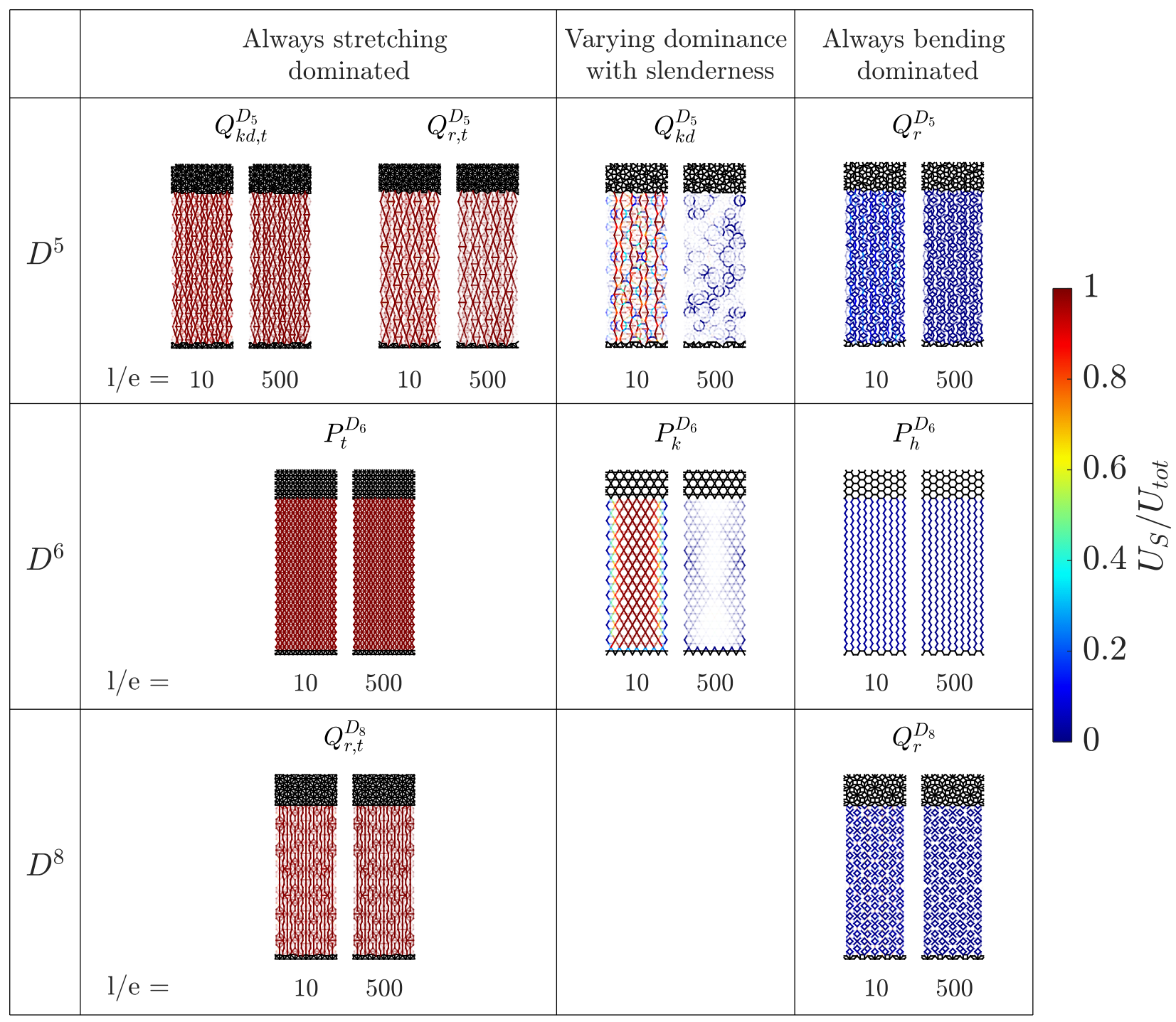

Figure 4: Spatial distribution of the strain energy in the different samples for slenderness equals to 10 and 500 . Transparency of the beams indicates the quantity of the energy stored, opaque beams store at least $25 \%$ of the maximum stored. Color specifies the tensile energy ratio.

\section{References}

[1] V. S. Deshpande, M. F. Ashby, N. A. Fleck, Acta Materialia 49 (2001) 1035-1040. doi:10.1016/ s1359-6454(00) 00379-7.

[2] B. Budiansky, International Journal of Solids and Structures 36 (1999) 3677-3708. doi:10.1016/ s0020-7683(98) 00169-3.

[3] O. Bouaziz, Y. Bréchet, J. Embury, Advanced Engineering Materials 10 (2008) 24-36. doi:10.1002/ adem. 200700289.

[4] J. B. Berger, H. N. G. Wadley, R. M. McMeeking, Nature 543 (2017) 533-537. doi:10.1038/nature21075.

[5] N. A. Fleck, V. S. Deshpande, M. F. Ashby, Proceedings of the Royal Society A: Mathematical, Physical and Engineering Sciences 466 (2010) 2495-2516. doi:10.1098/rspa.2010.0215.

[6] A. Glacet, J. Réthoré, A. Tanguy, F. Morestin, Scripta Materialia 156 (2018) 23-26. doi:10.1016/j. scriptamat.2018.07.001. 
[7] D. Shechtman, I. Blech, D. Gratias, J. W. Cahn, Physical Review Letters 53 (1984) 1951-1953. doi:10. 1103/physrevlett.53.1951.

[8] P. Gong, C.-Z. Hu, X. Zhou, L. Miao, X. Wang, The European Physical Journal B 52 (2006) 477-481. doi:10.1140/epjb/e2006-00323-6.

[9] Y. Wang, O. Sigmund, Extreme Mechanics Letters 34 (2020) 100596. doi:10.1016/j .eml .2019.100596.

[10] L. J. Gibson, M. F. Ashby, Cellular Solids, Cambridge University Press, 1997. doi:10.1017/ cbo9781139878326.

[11] V. S. Deshpande, N. A. Fleck, M. F. Ashby, Journal of the Mechanics and Physics of Solids 49 (2001) 1747-1769. doi:10.1016/s0022-5096(01)00010-2.

[12] M. Mazur, M. Leary, M. McMillan, S. Sun, D. Shidid, M. Brandt, in: Laser Additive Manufacturing, Elsevier, 2017, pp. 119-161. doi:10.1016/b978-0-08-100433-3.00005-1.

[13] M. F. Ashby, Philosophical Transactions of the Royal Society A: Mathematical, Physical and Engineering Sciences 364 (2005) 15 - 30. doi:10.1098/rsta.2005.1678.

[14] F. Albertini, J. Dirrenberger, A. Molotnikov, C. Sollogoub, Journal of Applied Mechanics 86 (2019). doi:10.1115/1.4044542.

[15] X. Badiche, S. Forest, T. Guibert, Y. Bienvenu, J.-D. Bartout, P. Ienny, M. Croset, H. Bernet, Materials Science and Engineering: A 289 (2000) 276-288. doi:10.1016/s0921-5093(00)00898-4.

[16] D. Levine, P. J. Steinhardt, Physical Review Letters 53 (1984) 2477-2480. doi:10.1103/physrevlett. 53.2477.

[17] T. Janssen, Physics Reports 168 (1988) 55-113. doi:10.1016/0370-1573(88) 90017-8.

[18] R. Penrose, in: Bulletin of the Institute of Mathematics and Its Applications, volume 10, pp. 266-271.

[19] R. Penrose, The Mathematical Intelligencer 2 (1979) 32-37. doi:10.1007/bf03024384.

[20] R. Ammann, B. Grünbaum, G. C. Shephard, Discrete \& Computational Geometry 8 (1992) 1-25. doi:10. $1007 / \mathrm{bf} 02293033$.

[21] F. P. M. Beenker, Algebraic theory of non-periodic tilings of the plane by two simple building blocks : a square and a rhombus, EUT report. WSK, Dept. of Mathematics and Computing Science, Eindhoven University of Technology, 1982.

[22] R. M. Christensen, Journal of Applied Mechanics 54 (1987) 772-777. doi:10.1115/1.3173115.

[23] N. Auffray, J. Dirrenberger, G. Rosi, International Journal of Solids and Structures 69-70 (2015) 195-206. doi:10.1016/j.ijsolstr.2015.04.036.

[24] T. Janssen, A. Janner, Acta Crystallographica Section B Structural Science, Crystal Engineering and Materials 70 (2014) 617-651. doi:10.1107/s2052520614014917.

[25] B. Grünbaum, G. C. Shephard, Tilings and Patterns, W. H. Freeman \& Co., USA, 1986.

[26] J. C. Maxwell, The London, Edinburgh, and Dublin Philosophical Magazine and Journal of Science 27 (1864) 294-299. 